\title{
Targeted nanoparticles containing the proresolving peptide Ac2-26 protect against advanced atherosclerosis in hypercholesterolemic mice
}

\section{Citation}

Fredman G., N. Kamaly, S. Spolitu, J. Milton, D. Ghorpade, R. Chiasson, G. Kuriakose, M. Perretti, O. Farokhzad, and I. Tabas. 2015. "Targeted Nanoparticles Containing the Proresolving Peptide Ac2-26 Protect Against Advanced Atherosclerosis in Hypercholesterolemic Mice." Science Translational Medicine 7 (275). doi:10.1126/scitranslmed.aaa1065.

\section{Published Version}

doi:10.1126/scitranslmed.aaa1065

\section{Permanent link}

http://nrs.harvard.edu/urn-3:HUL.InstRepos:29058532

\section{Terms of Use}

This article was downloaded from Harvard University's DASH repository, and is made available under the terms and conditions applicable to Open Access Policy Articles, as set forth at http:// nrs.harvard.edu/urn-3:HUL.InstRepos:dash.current.terms-of-use\#OAP

\section{Share Your Story}

The Harvard community has made this article openly available.

Please share how this access benefits you. Submit a story.

Accessibility 
Published in final edited form as:

Sci Transl Med. 2015 February 18; 7(275): 275ra20. doi:10.1126/scitranslmed.aaa1065.

\title{
Targeted nanoparticles containing the proresolving peptide Ac2-26 protect against advanced atherosclerosis in hypercholesterolemic mice
}

\author{
Gabrielle Fredman ${ }^{\# 1}$, Nazila Kamaly ${ }^{\# 2}$, Stefano Spolitu ${ }^{1}$, Jaclyn Milton ${ }^{2}$, Devram \\ Ghorpade $^{1}$, Raymond Chiasson ${ }^{2}$, George Kuriakose ${ }^{1}$, Mauro Perretti ${ }^{3}$, Omid Farokzhad ${ }^{2, \dagger}$, \\ and Ira Tabas ${ }^{1, \dagger}$ \\ ${ }^{1}$ Departments of Medicine, Pathology \& Cell Biology, and Physiology, Columbia University, New \\ York, NY 10032, USA \\ ${ }^{2}$ Laboratory of Nanomedicine and Biomaterials, Department of Anesthesiology, Brigham and \\ Women's Hospital, Harvard Medical School, Boston, MA 02115, USA \\ ${ }^{3}$ The William Harvey Research Institute, Barts and The London School of Medicine, Charterhouse \\ Square, London EC1M 6BQ, UK \\ \# These authors contributed equally to this work.
}

\begin{abstract}
Chronic, nonresolving inflammation is a critical factor in the clinical progression of advanced atherosclerotic lesions. In the normal inflammatory response, resolution is mediated by several agonists, among which is the glucocorticoid-regulated protein called annexin A1. The proresolving actions of annexin A1, which are mediated through its receptor $N$-formyl peptide receptor 2 (FPR2/ALX), can be mimicked by an amino-terminal peptide encompassing amino
\end{abstract}

\footnotetext{
Copyright 2015 by the American Association for the Advancement of Science; all rights reserved.

${ }^{\dagger}$ Corresponding authors. iatl@ columbia.edu (I.T.); ofarokhzad@ zeus.bwh.harvard.edu (O.F.) .

Author contributions: G.F., N.K., O.F., and I.T. conceived, designed, and supervised the research and wrote the manuscript; N.K., J.M., and R.C. designed, engineered, and optimized the nanoparticle technologies used in this publication; S.S., D.G., and G.K. conducted lesional analysis experiments; M.P. provided $\mathrm{Fpr}^{-/-}$femurs, provided advice for the experiments, and helped write the manuscript.

SUPPLEMENTARY MATERIALS

www.sciencetranslationalmedicine.org/cgi/content/full/7/275/275ra20/DC1

Fig. S1. Engineered NPs with Col IV targeting peptide.

Fig. S2. Metabolic parameters of the mice were unaffected by treatment with Col IV-Ac2-26 NPs or controls.

Fig. S3. Col IV-Ac2-26 NP treatment does not affect collagen content, superoxide, or mRNA of IllO or Acta2 in the liver or spleen.

Fig. S4. Col IV-Ac2-26 NP treatment does not affect aortic root lesion area or the numbers of lesional macrophages and SMCs.

Fig. S5. Ac2-26 decreases 7KC-induced ROS in an FPR2/ALX- and Nox2-dependent manner.

Fig. S6. Lesion FPR2/ALX decreases as atherosclerosis progresses.

Fig. S7. Metabolic parameters of the bone marrow transplant mice described in Fig. 4.

Competing interests: An international patent application has been filed for the inflammation-resolving NPs (WO 2014/145749 A1). In compliance with the Brigham and Women's Hospital and Harvard Medical School institutional guidelines, O.F. discloses his financial interest in BIND Biosciences, Selecta Biosciences, and Blend Therapeutics, three biotechnology companies developing NP technologies for medical applications. BIND, Selecta, and Blend did not support the research in this study, and currently, these companies have no rights to any technology or intellectual property developed as part of this research.

Data and materials availability: All reasonable requests for collaboration involving materials used in the research will be fulfilled provided that a written agreement is executed in advance between Brigham and Women's Hospital or Columbia University and the requester (and his or her affiliated institution). Such inquiries or requests for additional data should be directed to the corresponding authors.
} 
acids 2-26 (Ac2-26). Collagen IV (Col IV)-targeted nanoparticles (NPs) containing Ac2-26 were evaluated for their therapeutic effect on chronic, advanced atherosclerosis in fat-fed $\mathrm{Ldll}^{-1-}$ mice. When administered to mice with preexisting lesions, Col IV-Ac2-26 NPs were targeted to lesions and led to a marked improvement in key advanced plaque properties, including an increase in the protective collagen layer overlying lesions (which was associated with a decrease in lesional collagenase activity), suppression of oxidative stress, and a decrease in plaque necrosis. In mice lacking FPR2/ALX in myeloid cells, these improvements were not seen. Thus, administration of a resolution-mediating peptide in a targeted NP activates its receptor on myeloid cells to stabilize advanced atherosclerotic lesions. These findings support the concept that defective inflammation resolution plays a role in advanced atherosclerosis, and suggest a new form of therapy.

\section{INTRODUCTION}

Atherosclerosis, the disease process responsible for the leading cause of death in the industrialized world, is driven by a chronic, maladaptive inflammatory response (1). This response is triggered by the persistent and amplified subendothelial retention of apolipoprotein B-containing lipoproteins in focal areas of the arterial tree (2). The normal inflammatory response is followed by a resolution phase, which is effected by specific lipid and protein mediators that terminate inflammation and promote tissue repair $(3,4)$. In this regard, many of the characteristics of clinically dangerous advanced plaques, including thinning of a protective "cap" of subendothelial collagen, oxidative stress, defective clearance of dead cells (efferocytosis), and tissue-damaging necrosis, suggest that this resolution process is defective in these lesions $(5,6)$. However, more proof in vivo is needed to support this concept. In addition, cell-specific resolution processes that fail in advanced atherosclerosis need to be identified, and the therapeutic potential of enhancing resolution in this disease remains to be explored.

The therapeutic potential of enhancing resolution in a chronic disease like atherosclerosis could be substantial in that mediators of resolution, unlike anti-inflammatory drugs per se, have a much greater potential to suppress inflammation and promote tissue repair without compromising host defense $(7,8)$.

Although atherosclerosis can be treated with low-density lipoprotein (LDL) lowering by statins, cardiovascular disease remains the leading cause of death worldwide (9). In view of the evidence for defective inflammation resolution in atherosclerosis $(5,6)$, proresolving therapy may be able to expand treatment options for cardiovascular disease. To date, mouse studies have shown the benefit of adenoviral interleukin-10 (IL-10) treatment (10), but systemic delivery of IL-10 would require frequent dosing at a high dose, which is expensive, inconvenient, and potentially dangerous, especially for a chronic condition like atherosclerosis. For example, adverse effects of high-dose systemic recombinant IL-10 therapy in Crohn's disease patients include thrombocytopenia; anemia; proliferation of B cells, CD8 ${ }^{+}$cytotoxic $\mathrm{T}$ cells, natural killer cells, and mast cells; and elevated interferon- $\gamma$ $(11,12)$. Moreover, systemic IL-10 can also compromise host defense, which can be avoided by tissue-specific transgenesis in mice (13). 
One class of resolution mediators consists of fatty acid-derived lipids called lipoxins, resolvins, protectins, and maresins, which are collectively referred to as specialized proresolving lipid mediators (3). Several of these mediators, notably RvE1, have shown benefit in preclinical models of certain chronic inflammatory diseases, including asthma, rheumatoid arthritis, and periodontal disease $(14,15)$. In humans, proresolving lipid mediators have shown benefit in dry eye syndrome, a chronic inflammatory disease affecting the ocular surface (www.clinicaltrials.gov; NCT00941018). Another class of proresolving mediators includes proteins such as transforming growth factor- $\beta$ (TGF- $\beta$ ) and annexin A1 (4). Endogenous annexin A1, a 37-kD protein, mediates inflammation resolution in several disease models, and administration of a 25-amino acid peptide encompassing its pharmacophore N-terminal region, called Ac2-26, can mimic the effects of annexin A1 (16). Annexin A1 and Ac2-26 bind and activate a specific G protein-coupled receptor (GPCR) called $N$-formyl peptide receptor 2 (FPR2/ALX) to evoke their protective actions, and intriguingly, this is the same receptor used by two lipid mediators: resolvin D1 (RvD1) and lipoxin $\mathrm{A}_{4}\left(\mathrm{LXA}_{4}\right)(17-20)$.

We chose the proresolving mediator Ac2-26 because it is a small, stable peptide and can be targeted to atherosclerotic lesions using nanotechnology. Ac2-26 is rapidly cleared from plasma, and so repeated administrations of large amounts of peptide are needed for efficacy (21). Although this strategy may be acceptable for the treatment of acute inflammatory conditions, it would be difficult to use in chronic diseases such as atherosclerosis. In an attempt to overcome this problem, we recently encapsulated Ac2-26 in controlled release polymeric nanoparticles (NPs) that are targeted to sites of injury through the addition of a collagen IV (Col IV)-binding peptide (22). As initial proof of concept, we showed that these Col IV-Ac2-26 NPs accumulated in areas of acute inflammation and tissue injury and enhanced resolution to a much greater extent than free Ac2-26 peptide. However, these acute settings are very different from chronic, nonresolving inflammatory conditions because of the central role of failed resolution in the chronic conditions and the need for prolonged therapy.

In this context, we now report our findings on the application of this strategy to advanced atherosclerosis. Because Col IV is a major component of the vascular basement membrane that becomes exposed at sites of vascular injury and inflammation $(23,24)$, we reasoned that Col IV exposure at sites of atherosclerosis would facilitate lesional targeting of the NPs. In mice, we show that Col IV-Ac2-26 NPs home to atherosclerotic lesions and stabilize vulnerable plaques by reducing lesional superoxide and collagenase activity in a myeloidFPR2/ALX-dependent manner. These findings are consistent with the concept that inflammation resolution is defective in advanced atherosclerosis. Further, these findings suggest a new modality to combat inflammation in atherosclerosis in a way that is predicted to compromise the host defense system less than directly targeting inflammatory mediators or their receptors. 


\section{RESULTS}

\section{Col IV-Ac2-26 NPs home to experimental atherosclerotic lesions}

The design of the Col IV-Ac2-26 NPs used in this study was based on four principles (22):

(i) long circulating half-life to facilitate localization and plaque accumulation; (ii) small size to facilitate permeation of the endothelium, that is, NPs that are $\sim 100 \mathrm{~nm}$ or smaller can access the vascular wall by enhanced permeability and retention; (iii) molecular targeting to enhance retention in the plaque; and (iv) controlled, extracellular cargo release to enable the drug to activate its cell surface receptor target at an optimal concentration and for a maximal duration. The NPs were composed of PLGA and PEG, and were conjugated to Ac2-26 peptide (fig. S1). Both the Ac2-26 NPs and control NPs with scrambled peptide were formulated using nanoprecipitation such that the diameter was $<100 \mathrm{~nm}$ and the surface charge was $<-30 \mathrm{mV}$ (fig. S1, A and D), as described previously (22). The surface of the NPs was decorated with a Col IV-binding heptapeptide, which we identified previously from a phage display library (23). Control therapy was Ac2-26 NPs that lacked the Col IVbinding peptide.

To test targeting of atherosclerotic lesions, $\mathrm{Ldlr}^{-/-}$mice were fed the Western diet for 12 weeks to promote atherosclerosis and then injected intravenously with Ac2-26 NPs or Col IV-Ac2-26 NPs conjugated with Alexa 647. Because the NPs were designed to release $>95 \%$ of the Ac2-26 over 5 days, we chose to harvest the aortic roots, spleens, and livers 5 days after NP infusion. We found that Alexa 647 fluorescence in the aortic root was twofold greater for Col IV-Ac2-26 NPs than for the Ac2-26 NPs, particularly in the subendothelial region (Fig. 1, A and B). About 70\% of the Col IV-Ac2-26 NPs were present in the atherosclerotic lesions relative to spleen and liver, whereas the opposite relative distribution was found for the non-Col IV NPs (Fig. 1B). Thus, Col IV-Ac2-26 NPs bind more selectively to atherosclerotic lesions than do nontargeted Ac2-26 NPs.

\section{Col IV-Ac2-26 NPs increase subendothelial collagen in mice with established atherosclerosis}

To test the efficacy of Col IV-Ac2-26 NPs in advanced atherosclerosis, $\mathrm{Ldlr}^{-/-}$mice were fed a Western diet for 12 weeks, after which Col IV-Ac2-26 NPs, free Ac2-26, control Col IV-scrambled peptide NPs, and vehicle control (normal saline) were injected intravenously once per week over a 5-week period. Body weights, plasma cholesterol, lipoprotein profiles, and fasting blood glucose were similar between the four groups of mice (fig. S2). Aortic root and brachiocephalic (BCA) lesions were harvested, and their cross sections were analyzed for several hallmarks of advanced plaque progression that are associated with vulnerable plaques in humans (25). One such hallmark is thinning of a protective layer, or cap, of subendothelial collagen that overlies the necrotic center of advanced lesions.

As expected for $\mathrm{Ldlr}^{-/-}$mice fed the Western diet for 17 weeks, the aortic root lesions of the control cohort had relatively thin layers of subendothelial collagen (Fig. 2A). In contrast, this collagen layer was significantly thicker in the Col IV-Ac2-26 NP-treated group compared with the other groups, and comparison of 12 weeks (before treatment) versus 17 weeks indicated that this effect of the Col IV-Ac2-26 NPs was associated with an increase 
in cap thickness during the 5-week treatment period. In the spleen and liver, the amount of collagen and the expression of the fibrosis marker a-actin were unaffected by Col IVAc2-26 NPs (fig. S3), suggesting that the increase in collagen in the aortic root was a targeted process and not associated with potentially adverse fibrosis in other tissues. Overall lesion area and the numbers of lesional macrophages and smooth muscle cells (SMCs) were similar among all treatment groups (fig. S4, A to C), consistent with a primary effect of the Col IV-Ac2-26 NPs on the critical plaque parameter of lesion remodeling.

One likely cause of fibrous cap thinning is increased activity of collagen-degrading metalloproteinases, which are secreted by inflammatory macrophages $(26,27)$. Therefore, we tested whether the increase in fibrous cap thickness in the aortic root lesions of Col IVAc2-26 NP-treated mice was associated with a decrease in lesional collagenase activity. Using lesional cross sections incubated with a fluorescently labeled substrate that increases in fluorescence upon hydrolysis, we found that collagenase activity was significantly lower in the lesions of Col IV-Ac2-26 NP-treated mice (Fig. 2B). Another possible mechanism for increased fibrous cap thickness could be enhanced collagen biosynthesis. Consistent with this possibility was the finding that the lesions of Col IV-Ac2-26 NP-treated mice had significantly enhanced Col3al mRNA (Fig. 2C). These combined data are consistent with the idea that Col IV-Ac2-26 NPs increase fibrous cap thickness by a combination of decreasing cap degradation and increasing cap synthesis.

\section{Col IV-Ac2-26 NPs suppress superoxide and increase IL-10 in atherosclerotic lesions}

Hydrogen peroxide and other reactive oxygen intermediates (ROIs) are generated during the acute inflammatory response to kill pathogens, but excessive ROIs can damage host tissues. Hence, a key function of the resolution response is to terminate the production of ROIs (28). One of the signs of defective inflammation resolution in advanced atherosclerosis is excessive oxidative stress $(29,30)$. Using aortic root sections incubated with dihydroethidium (DHE), a fluorescent probe that detects superoxide in tissues, we observed that lesions of Col IV-Ac2-26 NP-treated mice had significantly less superoxide than those of the other control groups (Fig. 3A). Superoxide was not decreased in the spleen and liver of Col IV-Ac2-26 NP-treated mice, further suggesting a tissue-specific action of these NPs at the aortic root (fig. S3).

To further probe the mechanism of the decrease in superoxide by Ac2-26, we studied primary macrophages incubated with an oxysterol found in atherosclerotic lesions, 7ketocholesterol (7KC), which is known to induce Nox2-dependent oxidative stress in macrophages (31). Consistent with the in vivo lesional data, Ac2-26 significantly blocked 7KC-induced reactive oxygen species (ROS) in a FPR2/ALX-dependent manner (fig. S5A). A parallel set of experiments revealed that $7 \mathrm{KC}$-induced ROS was markedly decreased in

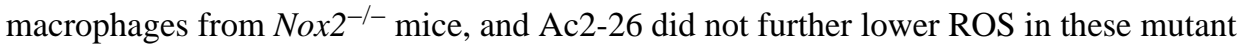
macrophages (fig. S5B). Although these data are difficult to interpret given the very low level of ROS in the $\mathrm{Nox}^{-/-}$macrophages treated with $7 \mathrm{KC}$ alone, it is possible that part of the mechanism of Ac2-26-mediated reduction in ROS is through suppression of 7KCinduced Nox 2 activation. 
Another source of ROS in atherosclerotic lesional macrophages is mitochondrial oxidative stress (mitoOS), which promotes plaque development by enhancing inflammation in lesions (30). We therefore investigated whether Col IV-Ac2-26 NPs temper mitoOS in lesions. MitoOS causes oxidative damage to mitochondrial DNA and thus can be assayed by measuring a marker of this process-mitochondrial (non-nuclear) 8-hydroxydeoxyguanosine (8-OHdG); this assay also measures nuclear 8-OHdG, which reflects more global intracellular oxidative stress (30). Col IV-Ac2-26 NPs significantly decreased both nuclear and nonnuclear 8-OHdG staining in lesional macrophages (Fig. 3, B and C), suggesting that the treatment tempered both general oxidative stress and mitoOS in lesional macrophages.

An important hallmark of inflammation resolution is increased production of proresolving mediators (32). We therefore assayed expression of the mRNA for the proresolving cytokine IL-10 and found that it was elevated in the lesions of Col IV-Ac2-26 NP-treated mice (Fig. 3D). $I l 10$ mRNA was not significantly increased in the spleen or liver of the Col IV-Ac2-26 NP-treated mice, again suggesting a tissue-targeted action of these NPs (fig. S3).

\section{Col IV-Ac2-26 NPs exert atheroprotective effects in myeloid-derived cells in an FPR2/ALX- dependent manner}

FPR2/ALX, the cell surface receptor for the proresolving ligands annexin A1, Ac2-26, $\mathrm{LXA}_{4}$, and RvD1, was present on $\sim 30 \%$ of murine $\mathrm{Mac}^{+}$macrophages and on $\sim 40 \%$ of Mac $3^{-}$cells at 8 and 12 weeks after starting a Western diet (fig. S6). The percentage of FPR2/ALX ${ }^{+} \mathrm{Mac}^{+}$and FPR2/ALX ${ }^{+} \mathrm{Mac}^{-}$cells significantly decreased after 17 weeks of Western diet, raising the possibility that a decrease in the level of macrophage FPR2/ALX contributes to defective inflammation in advanced atherosclerosis.

We determined whether the proresolving effects of Col IV-Ac2-26 NPs on atherosclerotic lesions were linked to the known mechanistic basis of Ac2-26 action, that is, binding to and activation of the FPR2/ALX receptor on myeloid-derived cells. Irradiated $\mathrm{Ldlr}^{-{ }^{-}}$mice were transplanted with bone marrow cells from wild-type (WT) or from $\mathrm{Fpr}^{-/-}$mice and then subjected to the NP treatment protocol and lesional analyses as in the previous studies. As with the non-transplant model, lesion area and systemic metabolic parameters were similar among the four groups of mice (figs. S4D and S7). In the WT-transplanted mice $(\mathrm{WT} \rightarrow \mathrm{WT}$ ), Col IV-Ac2-26 NPs enhanced fibrous cap thickness (Fig. 4A), similar to what was found in the non-BMT (bone marrow transplant) model. However, this response was blunted in $\mathrm{Fpr}^{-{ }_{-}}$-transplanted WT mice $\left(\mathrm{Fpr}^{-{ }_{-}} \rightarrow \mathrm{WT}\right)$. The trend toward increased cap thickness in the $\mathrm{Fpr}^{-/-} \rightarrow$ WT Ac2-26-NP group may reflect a modest, direct action of Ac2-26 on non-bone marrow-derived intimal SMCs, which are known to be a source of collagen in lesions, but this alternate mechanism was not investigated here.

Treatment with Col IV-Ac2-26 NPs also significantly reduced collagenase activity and lesional superoxide in the lesions of WT $\rightarrow$ WT mice, but not in the lesions of the $\mathrm{Fpr}^{-\mathrm{I}^{-}} \rightarrow$ WT mice (Fig. 4, B and C). Together, these data indicate that the protective actions of Col IV-Ac2-26 NPs were due primarily to activation of their FPR2/ALX receptor on myeloid-derived cells. 


\section{Col IV-Ac2-26 NPs decrease lesion area, necrotic area, and oxidative stress in brachiocephalic arterial plaques}

In mouse models of atherosclerosis, the brachiocephalic artery (BCA) tends to develop advanced necrotic plaques, perhaps consequent to the effects of disturbed blood flow on lesional endothelium (33). Inspection of the intact BCA, where atherosclerotic lesions appear white, as well as quantitative analysis of lesion cross sections revealed that Col IVAc2-26 NPs decreased lesion area and especially necrotic area, where the suppression was about $80 \%$ (Fig. 5, A to D). Indeed, the decrease in lesion area measurement in the BCA can be explained mostly by the marked decrease in necrotic area, because in these lesions, the necrotic area represents a substantial portion $(\sim 40 \%)$ of the total lesion area. We also quantified lesional intercellular adhesion molecule-1 (ICAM) immunostaining, a marker of inflamed vasculature, and found a substantial decrease in the cohort treated with Col IVAc2-26 NPs compared with animals treated with the scrambled control peptide (Fig. 5E). Finally, as with the aortic root, lesional DHE staining was markedly less in the BCA of Col IV-Ac2-26 NP-treated mice (Fig. 5F).

\section{DISCUSSION}

The goal of this study was to therapeutically target defective inflammation resolution in advanced atherosclerotic plaques and thereby suppress plaque progression. To this end, we administered a mediator of inflammation resolution, Ac2-26, in an NP form, specifically targeting Col IV in atherosclerotic plaques. We demonstrated thickening of the plaque fibrous cap, suppression of lesional superoxide, and, in BCA lesions, a decrease in plaque necrosis. Each of these improvements is consistent with improved resolution of inflammation.

Previous studies have used various genetic manipulations to overexpress a proresolving cytokine, IL-10, in mouse models of atherosclerosis. In one such study, $\mathrm{Ldlr}^{-/-}$mice were transplanted with the bone marrow of IL-10 transgenic mice, and aortic root plaque complexity was decreased in these chimeric mice compared with lesions of control $\mathrm{WT} \rightarrow \mathrm{Ldlr}^{-/}$mice (34). Despite these promising findings, whole-organism genetic manipulation is not a therapeutic option for humans, and the use of cytokines for atherosclerosis would need to be adapted to a therapeutic approach. The Col IV-Ac2-26 NPs in this study led to an increase in 1110 mRNA in lesions, suggesting that its benefits may, in part, be mediated by IL-10. Ac2-26 targets the receptor FPR2/ALX, and a key attribute of such ligands is that they dampen excessive inflammation without compromising host defense (3). This is critical because therapies that target inflammatory cytokines often compromise host defense against pathogens, particularly when they are administered over a long period of time (8). Thus, therapeutics that enhance resolution might have a better benefit/risk ratio than currently available anti-inflammatory drugs, such as anti-TNFa (tumor necrosis factor-a) (8).

A key set of questions that emerges from this study is related to the molecular and cellular mechanisms that underlie the protective effects of Ac2-26 and FPR2/ALX signaling. For example, the increase in fibrous cap thickness was associated with a decrease in lesional collagenase activity and an increase in Col3al expression, but the mechanism and 
importance of these processes in fibrous cap remodeling remain to be elucidated. Regarding collagen synthesis, annexin A1 can act directly on SMCs (35). However, the results of the $\mathrm{Fpr}^{-/-}$BMT study raise the possibility that Ac2-26 enhances the production of collagen by lesional SMCs by first inducing macrophages to secrete TGF- $\beta$, which is a profibrotic mediator that can promote plaque stabilization (36). Another question is how the intralesional location of collagen accrual is regulated by proresolving mediators. Although collagen near the lumen in advanced plaques is beneficial to prevent rupture, collagen accrual in other parts of the lesions may promote detrimental vascular stiffness (37). Other examples of mechanistic questions for future investigation include how Ac2-26 quenches oxidative stress and how it suppresses plaque necrosis (38-40). Finally, another FPR2/ALX ligand, RvD1, can decrease the ratio of leukotriene to lipoxin in leukocytes in acute peritonitis $(41,42)$; if this process occurs in lesional macrophages, it could provide a mechanism for decreased influx of inflammatory leukocytes, because leukotriene $\mathrm{B}_{4}\left(\mathrm{LTB}_{4}\right)$ has chemoattractant activity. Moreover, if lesional $\mathrm{LXA}_{4}$ levels were increased by Col IVAc2-26 NPs, this may amplify the Ac2-26 effect, because $\mathrm{LXA}_{4}$ activates the FPR2/ALX receptor and stimulates annexin A1 mobilization from neutrophils (31).

This study raises the question as to why resolution becomes defective as lesions progress. There is evidence that endogenous resolution pathways are operational in earlier lesions, notably those activated by IL-10 and 12/15-lipoxygenase (LOX)-derived specialized proresolving lipid mediators, such as $\mathrm{LXA}_{4}$ and $\mathrm{RvD1}(5,43,44)$. Possible explanations for why these and other pathways fail in advanced lesions include defective mediator production, enhanced mediator catabolism, conversion to inactive or proinflammatory compounds, and/or defective resolution receptor signaling. High-fat diet may also suppress the synthesis of proresolving mediators by 12/15-LOX (43). Furthermore, various specialized proresolving lipid mediators are converted into inactive forms through enzymatic metabolism $(45,46)$. Because FPR2/ALX receptor expression decreases as atherosclerosis advances, there is a possibility that therapeutics that target this receptor, such as Col IV-Ac2-26 NPs, are less effective when lesions reach this very advanced stage. However, the therapeutic effects of Col IV-Ac2-26 NPs in mice with advanced atherosclerosis shown here suggest that the residual receptor level is sufficient to mediate beneficial effects, at least when the ligand is presented in a high enough concentration. In contrast, the diminished FPR2/ALX expression during advanced atherosclerosis may not be sufficient for endogenous ligands to mount proresolving pathways, which may be an underlying mechanism contributing to defective resolution in advanced atherosclerosis.

Most atherosclerotic lesions do not progress and do not cause acute coronary syndromes, but those that do progress are responsible for the leading cause of death in the industrialized world: cardiovascular disease. Clinically important, unstable plaques in humans are not necessarily larger, but do have distinct morphological features that promote plaque rupture: larger necrotic cores, thinner fibrous caps, higher oxidative stress, and inflammation (25). Therefore, a critical goal in this area of research is to identify specific molecular-cellular mechanisms that lead to these plaque features and then, with this knowledge, devise strategies to therapeutically inhibit these processes in an effective and safe manner. Although the initial inflammatory response itself is an important process in plaque 
progression, directly inhibiting this response in a chronic setting, for example, through anticytokine therapy, runs the danger of compromising host defense. On the other hand, defective inflammation resolution accurately defines vulnerable plaque characteristics, and it is amenable to a therapeutic approach — targeted administration of resolution mediatorsthat is predicted not to compromise host defense $(7,8,16)$.

The experiments herein used one animal model of advanced atherosclerosis and tested a proof-of-concept targeted NP with one type of proresolving mediator. To bring targeted resolution mediator nanotherapeutics to the clinic for patients at high risk for atherothrombotic vascular events, additional confirmatory studies will be needed, including evaluation in more predictive models, such as fat-fed pigs and non-human primates. It is plausible that the properties of the NPs including the active proresolving agent will need to be optimized for suitable clinical applications (47). Although Ac2-26 has provided a useful proof-of-principle approach, other types of resolution mediators, including variations on the Ac2-26 peptide itself, may be more potent in promoting plaque resolution. Finally, detailed toxicity studies will be needed to show the safety of both the NP material and the resolution mediator cargo. With these future goals in mind, it is encouraging to note that NP therapeutics are now in clinical use or under clinical investigation for a myriad of other conditions including cancers (48), vaccines (49), and imaging (50). Thus, our atheroprotective strategy that capitalizes on plaque targeting to deliver a proresolving mediator may hold promise to decreasing the incidence of acute coronary events in subjects at high risk for atherothrombotic disease.

\section{MATERIALS AND METHODS}

\section{Study design}

The aim of this study was to test the therapeutic efficacy of targeted NPs containing the proresolving peptide Ac2-26 in experimental atherosclerosis. Animal experiments were conducted in $\mathrm{Ldlr}^{-/-}$mice using protocols approved by the Columbia University Standing Committee on Animals. The treatment groups included vehicle control, free Ac2-26 peptide, NPs containing a scrambled Ac2-26 peptide, and NPs containing Ac2-26 peptide. Unless otherwise indicated, 8 to 10 animals per group were investigated and were randomly assigned to the study groups. The experimentalists were blinded to the identity of the study groups while assaying the multiple endpoints in this study.

\section{Synthesis and NP formulation}

Copolymers of poly[lactic-co-glycolic acid- $b$-poly(ethylene glycol)] (PLGA-PEG), with or without Col IV-binding peptide or Alexa 647-cadaverine, were synthesized according to previously published methods (22) (fig. S1D). Briefly, the C terminus of 50:50 PLGA (Lactel, $43.4 \mathrm{kD}$ ) and the functional amino group of $\mathrm{NH}_{2}$-PEG (Laysan Bio Inc., 3400 daltons) were coupled using 1-ethyl-3-(3-dimethylaminopropyl)carbodiimide hydrochloride(EDC)and $N$-hydroxysuccinimide(NHS)(Sigma-Aldrich). Activated PLGANHS was coupled to the heterobifunctional maleimide-PEG-hydroxy to yield PLGA-PEGMal. The Col IV peptide-conjugated targeting polymer was then synthesized by conjugating KLWVLPK peptide to PLGA-PEG-Mal via the free thiol of the C-terminal GGGC linker 
using maleimide chemistry. The product was purified and washed by precipitation in cold methanol. A fluorescent polymer was also synthesized by coupling carboxy-terminated PLGA to Alexa 647-cadaverine.

The polymer ( $3 \mathrm{mg}$ ) was mixed with $120 \mu \mathrm{g}$ of Ac2-26

(AMVSEFLKQAWFIENEEQEYVQTVK) (Tocris Bioscience) or scrambled control peptide (WLKQKFQESVEQIAYVMENATEFEV) (Mimotopes) and dissolved in acetonitrile. This solution was then added dropwise to $10 \mathrm{ml}$ of nuclease-free water, stirred for 2 hours, and then passed through a sterile $0.45-\mu \mathrm{m}$ syringe filter. The NPs were then centrifuged at $3000 \mathrm{~g}$ for $20 \mathrm{~min}$ using an Amicon Ultra-15 centrifugal filter units (molecular weight cutoff, $100 \mathrm{kD}$; Sigma-Aldrich), washed with deionized water, resuspended in $1 \mathrm{ml}$ of nuclease-free $\mathrm{H}_{2} \mathrm{O}$, and then diluted with sterile saline before injection. The final composition of the fluorescently labeled Col IV-Ac2-26 NPs was 87\% PLGA-PEG-COOH, 5\% PLGA-PEG-Col IV peptide, 4\% PLGA-Alexa 647, and 4\% Ac2-26 (w/w).

\section{Animals and diets}

Male $\mathrm{Ldlr}^{-/-}$mice, 8 to 10 weeks old, were purchased from The Jackson Laboratory and placed on a Western-type diet (TD.88137, Harlan Teklad) for 12 weeks. After 12 weeks, the mice were continued on the Western diet for an additional 5 weeks and injected intravenously once per week during this period with $200 \mu \mathrm{l}$ of the following: Col IV-Ac2-26 NPs or Col IV-scrambled peptide NPs (10 $\mu$ g of peptide per injection and an equivalent polymer amount), free Ac2-26 (10 $\mu \mathrm{g}$ per injection), or sterile saline (vehicle control). All procedures were conducted in accordance with protocols approved by the Columbia University Standing Committee on Animals guidelines for animal care.

\section{Atherosclerotic lesion analysis}

For morphometric lesion analysis, sections were stained with Harris' H\&E. Total lesion and necrotic areas were quantified as described previously (30). For plaque necrosis, boundary lines were drawn around regions that were free of H\&E staining, and area measurements were obtained using image analysis software. Collagen staining was performed using picrosirius red as per the manufacturer's instructions (Polysciences Inc.). Because fibrous cap thickness can vary throughout the lesions, these values were assessed by averaging the thickness in micrometers of the cap in three separate regions of the lesions (that is, one measurement for each of the shoulder regions and one measurement in the middle of the lesion) and then expressing as AU of cap thickness/lesion area. Collagenase activity in lesions was assayed using the DQ Collagenase Assay Kit from Life Technologies according to the manufacturer's instructions. Collagenase was analyzed by calculating MFI per lesion using ImageJ software.

For detection of superoxide, the frozen lesion sections were incubated with $1 \mu \mathrm{M}$ DHE for $20 \mathrm{~min}$ at $37^{\circ} \mathrm{C}$ and then washed twice with phosphate-buffered saline (29). Sections were counterstained with DAPI and mounted with antifade reagent (ProLong Gold). For both collagenase and DHE procedures, images were taken on a Zeiss fluorescence microscope and analyzed using ImageJ software. For mitoOS measurements, frozen sections were stained sequentially at $4^{\circ} \mathrm{C}$ with anti-Mac3 (1:200; BD clone M3/84) and then anti-8-OHdG 
(1:200; AB5830; EMD Millipore). Sections were then counterstained with DAPI to identify nuclei. Data were quantified as the percentage of total $\mathrm{Mac}^{+}$cells that had 8-OHdG staining that did not overlap with DAPI, indicating nonnuclear 8-OHdG ${ }^{+}$macrophages (30).

For FPR2/ALX expression, aortic root lesions were stained sequentially at $4{ }^{\circ} \mathrm{C}$ with antiMac3 and then anti-FPR2 antibody (1:200, sc-66901; Santa Cruz Biotechnology). Sections were then counterstained with DAPI to identify nuclei. Data were quantified as the percentage of $\mathrm{ALX}^{+} \mathrm{Mac}^{+}$per total Mac3 ${ }^{+}$cells or the percentage of $\mathrm{ALX}^{+} \mathrm{Mac}^{-}$cells per total Mac3- cells.

\section{LCM, RNA amplification, and RT-qPCR}

Aortic root sections were subjected to laser capture using a PALM LCM machine as described previously (51). RNA was isolated using the RNeasy Micro Kit (Qiagen) and linearly amplified using the MessageAmp II aRNA Kit (Ambion). The purity of the RNA was estimated by measuring absorbance at 260 and $280 \mathrm{~nm}$ using a NanoDrop (Thermo Scientific), and RNA with an $A_{260 / 280}$ of $>1.8$ was used for complementary DNA (cDNA) synthesis. cDNA was synthesized using the SuperScript VILO cDNA Synthesis Kit (Invitrogen), and qPCR was conducted using a 7500 Real-Time PCR system (Applied Biosystems) and SYBR Green chemistry. The following specific primers were purchased from Qiagen: Acta2 (\#QT00140119), Il10 (\#QT00106169), and Col3al (\#QT01055516).

\section{Bone marrow transplantation}

Eight- to 10 -week-old male $\mathrm{Ldlr}^{-1-}$ mice were lethally irradiated using a cesium $\gamma$ source at a dose of 1000 rads 4 to 6 hours before transplantation (30). Bone marrow cells were collected from the femurs and tibias of donor WT and $\mathrm{Fpr}^{-\mathrm{I}^{-}}$mice by flushing with sterile medium and then injected intravenously into the recipient mice, as described previously (52). All animal procedures used in this study followed Columbia University's institutional guidelines. Six weeks after bone marrow transplantation (at 14 weeks of age), $\mathrm{Ldll}^{-/-}$mice were fed the Western diet and treated with NPs as above.

\section{Statistics}

All results are presented as means \pm SEM. $P$ values were calculated using the Student's $t$ test or one-way ANOVA with post hoc Tukey analysis for normally distributed data. For nonnormally distributed data, the Mann-Whitney rank sum test was used.

\section{Supplementary Material}

Refer to Web version on PubMed Central for supplementary material.

\section{Acknowledgments}

We thank the Confocal and Specialized Microscopy Core at Columbia University's Irving Cancer Research Center for assistance with lesional imaging.

Funding:This work was supported by a Program of Excellence in Nanotechnology (PEN) Award, Contract \#HHSN268201000045C, from the NIH (O.F. and I.T.); NIH Pathway to Independence K99 grant HL119587 (G.F.); and the Welcome Trust Programme Grant (086867/Z/08). This work forms part of the research themes contributing to the translational research portfolio of Barts and The London NIHR Cardiovascular BRU (M.P.); 
NIH grants CA151884 and the David Koch-Prostate Cancer Foundation Award in Nanotherapeutics (O.F.); and NIH grants HL106019, HL075662, and HL054591 (I.T.).

\section{REFERENCES AND NOTES}

1. Libby P. Mechanisms of acute coronary syndromes and their implications for therapy. N. Engl. J. Med. 2013; 368:2004-2013. [PubMed: 23697515]

2. Tabas I, Williams KJ, Boren J. Subendothelial lipoprotein retention as the initiating process in atherosclerosis: Update and therapeutic implications. Circulation. 2007; 116:1832-1844. [PubMed: 17938300]

3. Buckley CD, Gilroy DW, Serhan CN. Proresolving lipid mediators and mechanisms in the resolution of acute inflammation. Immunity. 2014; 40:315-327. [PubMed: 24656045]

4. Perretti M, D'Acquisto F. Annexin A1 and glucocorticoids as effectors of the resolution of inflammation. Nat. Rev. Immunol. 2009; 9:62-70. [PubMed: 19104500]

5. Merched AJ, Ko K, Gotlinger KH, Serhan CN, Chan L. Atherosclerosis: Evidence for impairment of resolution of vascular inflammation governed by specific lipid mediators. FASEB J. 2008; 22:3595-3606. [PubMed: 18559988]

6. Tabas I. Macrophage death and defective inflammation resolution in atherosclerosis. Nat. Rev. Immunol. 2010; 10:36-46. [PubMed: 19960040]

7. Serhan CN, Brain SD, Buckley CD, Gilroy DW, Haslett C, O’Neill LA, Perretti M, Rossi AG, Wallace JL. Resolution of inflammation: State of the art, definitions and terms. FASEB J. 2007; 21:325-332. [PubMed: 17267386]

8. Tabas I, Glass CK. Anti-inflammatory therapy in chronic disease: Challenges and opportunities. Science. 2013; 339:166-172. [PubMed: 23307734]

9. Smith SC Jr. Collins A, Ferrari R, Holmes DR Jr. Logstrup S, McGhie DV, Ralston J, Sacco RL, Stam H, Taubert K, Wood DA, Zoghbi WA. Our time: A call to save preventable death from cardiovascular disease (heart disease and stroke). Circulation. 2012; 126:2769-2775. [PubMed: 22988010]

10. Liu Y, Li D, Chen J, Xie J, Bandyopadhyay S, Zhang D, Nemarkommula AR, Liu H, Mehta JL, Hermonat PL. Inhibition of atherogenesis in LDLR knockout mice by systemic delivery of adenoassociated virus type 2-hIL-10. Atherosclerosis. 2006; 188:19-27. [PubMed: 16300768]

11. Mosser DM, Zhang X. Interleukin-10: New perspectives on an old cytokine. Immunol. Rev. 2008; 226:205-218. [PubMed: 19161426]

12. Tilg H, van Montfrans C, van den Ende A, Kaser A, van Deventer SJ, Schreiber S, Gregor M, Ludwiczek O, Rutgeerts P, Gasche C, Koningsberger JC, Abreu L, Kuhn I, Cohard M, LeBeaut A, Grint P, Weiss G. Treatment of Crohn's disease with recombinant human interleukin 10 induces the proinflammatory cytokine interferon $\gamma$. Gut. 2002; 50:191-195. [PubMed: 11788558]

13. Oberholzer A, Oberholzer C, Moldawer LL. Interleukin-10: A complex role in the pathogenesis of sepsis syndromes and its potential as an anti-inflammatory drug. Crit. Care Med. 2002; 30:S58S63.

14. Hasturk H, Kantarci A, Ohira T, Arita M, Ebrahimi N, Chiang N, Petasis NA, Levy BD, Serhan CN, Van Dyke TE. RvE1 protects from local inflammation and osteoclast-mediated bone destruction in periodontitis. FASEB J. 2006; 20:401-403. [PubMed: 16373400]

15. Haworth O, Cernadas M, Yang R, Serhan CN, Levy BD. Resolvin E1 regulates interleukin 23, interferon $-\gamma$ and lipoxin $\mathrm{A}_{4}$ to promote the resolution of allergic airway inflammation. Nat. Immunol. 2008; 9:873-879. [PubMed: 18568027]

16. Perretti M, Dalli J. Exploiting the Annexin A1 pathway for the development of novel antiinflammatory therapeutics. Br. J. Pharmacol. 2009; 158:936-946. [PubMed: 19845684]

17. Cooray SN, Gobbetti T, Montero-Melendez T, McArthur S, Thompson D, Clark AJ, Flower RJ, Perretti M. Ligand-specific conformational change of the G-protein-coupled receptor ALX/FPR2 determines proresolving functional responses. Proc. Natl. Acad. Sci. U.S.A. 2013; 110:1823218237. [PubMed: 24108355] 
18. Perretti M, Chiang N, La M, Fierro IM, Marullo S, Getting SJ, Solito E, Serhan CN. Endogenous lipid- and peptide-derived anti-inflammatory pathways generated with glucocorticoid and aspirin treatment activate the lipoxin $\mathrm{A}_{4}$ receptor. Nat. Med. 2002; 8:1296-1302. [PubMed: 12368905]

19. Fiore S, Maddox JF, Perez HD, Serhan CN. Identification of a human cDNA encoding a functional high affinity lipoxin A4 receptor. J. Exp. Med. 1994; 180:253-260. [PubMed: 8006586]

20. Krishnamoorthy S, Recchiuti A, Chiang N, Yacoubian S, Lee CH, Yang R, Petasis NA, Serhan CN. Resolvin D1 binds human phagocytes with evidence for proresolving receptors. Proc. Natl. Acad. Sci. U.S.A. 2010; 107:1660-1665. [PubMed: 20080636]

21. Perretti M, Ahluwalia A, Harris JG, Goulding NJ, Flower RJ. Lipocortin-1 fragments inhibit neutrophil accumulation and neutrophil-dependent edema in the mouse. A qualitative comparison with an anti-CD11b monoclonal antibody. J. Immunol. 1993; 151:4306-4314. [PubMed: 8409403]

22. Kamaly N, Fredman G, Subramanian M, Gadde S, Pesic A, Cheung L, Fayad ZA, Langer R, Tabas I, Farokhzad OC. Development and in vivo efficacy of targeted polymeric inflammation-resolving nanoparticles. Proc. Natl. Acad. Sci. U.S.A. 2013; 110:6506-6511. [PubMed: 23533277]

23. Chan JM, Zhang L, Tong R, Ghosh D, Gao W, Liao G, Yuet KP, Gray D, Rhee JW, Cheng J, Golomb G, Libby P, Langer R, Farokhzad OC. Spatiotemporal controlled delivery of nanoparticles to injured vasculature. Proc. Natl. Acad. Sci. U.S.A. 2010; 107:2213-2218. [PubMed: 20133865]

24. Kalluri R. Basement membranes: Structure, assembly and role in tumour angiogenesis. Nat. Rev. Cancer. 2003; 3:422-433. [PubMed: 12778132]

25. Virmani R, Burke AP, Farb A, Kolodgie FD. Pathology of the unstable plaque. Prog. Cardiovasc. Dis. 2002; 44:349-356. [PubMed: 12024333]

26. Libby P. Collagenases and cracks in the plaque. J. Clin. Invest. 2013; 123:3201-3203. [PubMed: 23908120]

27. Moore KJ, Tabas I. Macrophages in the pathogenesis of atherosclerosis. Cell. 2011; 145:341-355. [PubMed: 21529710]

28. Serhan CN, Chiang N, Van Dyke TE. Resolving inflammation: Dual anti-inflammatory and proresolution lipid mediators. Nat. Rev. Immunol. 2008; 8:349-361. [PubMed: 18437155]

29. Liao X, Sluimer JC, Wang Y, Subramanian M, Brown K, Pattison JS, Robbins J, Martinez J, Tabas I. Macrophage autophagy plays a protective role in advanced atherosclerosis. Cell Metab. 2012; 15:545-553. [PubMed: 22445600]

30. Wang Y, Wang GZ, Rabinovitch PS, Tabas I. Macrophage mitochondrial oxidative stress promotes atherosclerosis and nuclear factor- $\kappa \mathrm{B}-$ mediated inflammation in macrophages. Circ. Res. 2014; 114:421-433. [PubMed: 24297735]

31. Brancaleone V, Dalli J, Bena S, Flower RJ, Cirino G, Perretti M. Evidence for an antiinflammatory loop centered on polymorphonuclear leukocyte formyl peptide receptor 2/lipoxin $\mathrm{A}_{4}$ receptor and operative in the inflamed microvasculature. J. Immunol. 2011; 186:4905-4914. [PubMed: 21398608]

32. Libby P, Tabas I, Fredman G, Fisher EA. Inflammation and its resolution as determinants of acute coronary syndromes. Circ. Res. 2014; 114:1867-1879. [PubMed: 24902971]

33. Gimbrone MA Jr. Garcia-Cardena G. Vascular endothelium, hemodynamics, and the pathobiology of atherosclerosis. Cardiovasc. Pathol. 2013; 22:9-15. [PubMed: 22818581]

34. Pinderski LJ, Fischbein MP, Subbanagounder G, Fishbein MC, Kubo N, Cheroutre H, Curtiss LK, Berliner JA, Boisvert WA. Overexpression of interleukin-10 by activated T lymphocytes inhibits atherosclerosis in LDL receptor-deficient Mice by altering lymphocyte and macrophage phenotypes. Circ. Res. 2002; 90:1064-1071. [PubMed: 12039795]

35. Viiri LE, Full LE, Navin TJ, Begum S, Didangelos A, Astola N, Berge RK, Seppala I, Shalhoub J, Franklin IJ, Perretti M, Lehtimaki T, Davies AH, Wait R, Monaco C. Smooth muscle cells in human atherosclerosis: Proteomic profiling reveals differences in expression of Annexin A1 and mitochondrial proteins in carotid disease. J. Mol. Cell. Cardiol. 2013; 54:65-72. [PubMed: 23154128]

36. Mallat Z, Gojova A, Marchiol-Fournigault C, Esposito B, Kamate C, Merval R, Fradelizi D, Tedgui A. Inhibition of transforming growth factor- $\beta$ signaling accelerates atherosclerosis and induces an unstable plaque phenotype in mice. Circ. Res. 2001; 89:930-934. [PubMed: 11701621] 
37. Libby P, Ridker PM, Hansson GK. Progress and challenges in translating the biology of atherosclerosis. Nature. 2011; 473:317-325. [PubMed: 21593864]

38. Peshavariya HM, Taylor CJ, Goh C, Liu GS, Jiang F, Chan EC, Dusting GJ. Annexin peptide Ac2-26 suppresses TNFa-induced inflammatory responses via inhibition of Rac1-dependent NADPH oxidase in human endothelial cells. PLOS One. 2013; 8:e60790. [PubMed: 23637767]

39. Perretti M, Croxtall JD, Wheller SK, Goulding NJ, Hannon R, Flower RJ. Mobilizing lipocortin 1 in adherent human leukocytes downregulates their transmigration. Nat. Med. 1996; 2:1259-1262. [PubMed: 8898757]

40. Maderna P, Cottell DC, Toivonen T, Dufton N, Dalli J, Perretti M, Godson C. FPR2/ALX receptor expression and internalization are critical for lipoxin $\mathrm{A}_{4}$ and annexin-derived peptide-stimulated phagocytosis. FASEB J. 2010; 24:4240-4249. [PubMed: 20570963]

41. Norling LV, Dalli J, Flower RJ, Serhan CN, Perretti M. Resolvin D1 limits polymorphonuclear leukocyte recruitment to inflammatory loci: Receptor-dependent actions. Arterioscler. Thromb. Vasc. Biol. 2012; 32:1970-1978. [PubMed: 22499990]

42. Fredman G, Ozcan L, Spolitu S, Hellman J, Spite M, Backs J, Tabas I. Resolvin D1 limits 5lipoxygenase nuclear localization and leukotriene $\mathrm{B}_{4}$ synthesis by inhibiting a calcium-activated kinase pathway. Proc. Natl. Acad. Sci. U.S.A. 2014; 111:14530-14535. [PubMed: 25246560]

43. Merched AJ, Serhan CN, Chan L. Nutrigenetic disruption of inflammation-resolution homeostasis and atherogenesis. J. Nutrigenet. Nutrigenomics. 2011; 4:12-24. [PubMed: 21474962]

44. Mallat Z, Besnard S, Duriez M, Deleuze V, Emmanuel F, Bureau MF, Soubrier F, Esposito B, Duez H, Fievet C, Staels B, Duverger N, Scherman D, Tedgui A. Protective role of interleukin-10 in atherosclerosis. Circ. Res. 1999; 85:e17-e24. [PubMed: 10521249]

45. Arita M, Oh SF, Chonan T, Hong S, Elangovan S, Sun YP, Uddin J, Petasis NA, Serhan CN. Metabolic inactivation of resolvin E1 and stabilization of its anti-inflammatory actions. J. Biol. Chem. 2006; 281:22847-22854. [PubMed: 16757471]

46. Sun YP, Oh SF, Uddin J, Yang R, Gotlinger K, Campbell E, Colgan SP, Petasis NA, Serhan CN. Resolvin D1 and its aspirin-triggered 17R epimer. Stereochemical assignments, anti-inflammatory properties, and enzymatic inactivation. J. Biol. Chem. 2007; 282:9323-9334. [PubMed: 17244615]

47. Kamaly N, Xiao Z, Valencia PM, Radovic-Moreno AF, Farokhzad OC. Targeted polymeric therapeutic nanoparticles: Design, development and clinical translation. Chem. Soc. Rev. 2012; 41:2971-3010. [PubMed: 22388185]

48. Bertrand N, Wu J, Xu X, Kamaly N, Farokhzad OC. Cancer nanotechnology: The impact of passive and active targeting in the era of modern cancer biology. Adv. Drug Deliv. Rev. 2014; 66:2-25. [PubMed: 24270007]

49. Zhao L, Seth A, Wibowo N, Zhao CX, Mitter N, Yu C, Middelberg AP. Nanoparticle vaccines. Vaccine. 2014; 32:327-337. [PubMed: 24295808]

50. Phillips E, Penate-Medina O, Zanzonico PB, Carvajal RD, Mohan P, Ye Y, Humm J, Gonen M, Kalaigian H, Schoder H, Strauss HW, Larson SM, Wiesner U, Bradbury MS. Clinical translation of an ultrasmall inorganic optical-PET imaging nanoparticle probe. Sci. Transl. Med. 2014; 6:260ra149.

51. Subramanian M, Thorp E, Hansson GK, Tabas I. Treg-mediated suppression of atherosclerosis requires MYD88 signaling in DCs. J. Clin. Invest. 2013; 123:179-188. [PubMed: 23257360]

52. Seimon TA, Nadolski MJ, Liao X, Magallon J, Nguyen M, Feric NT, Koschinsky ML, Harkewicz R, Witztum JL, Tsimikas S, Golenbock D, Moore KJ, Tabas I. Atherogenic lipids and lipoproteins trigger CD36-TLR2-dependent apoptosis in macrophages undergoing endoplasmic reticulum stress. Cell Metab. 2010; 12:467-482. [PubMed: 21035758] 
A

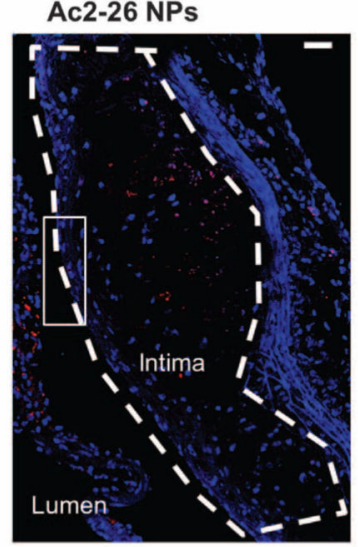

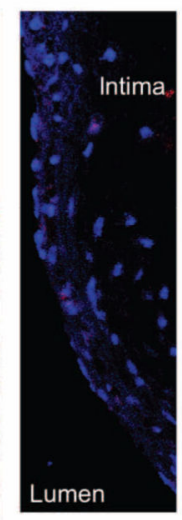

Col IV-Ac2-26 NPs

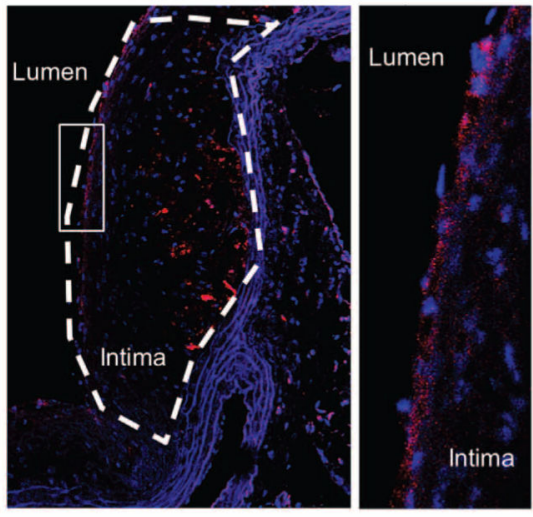

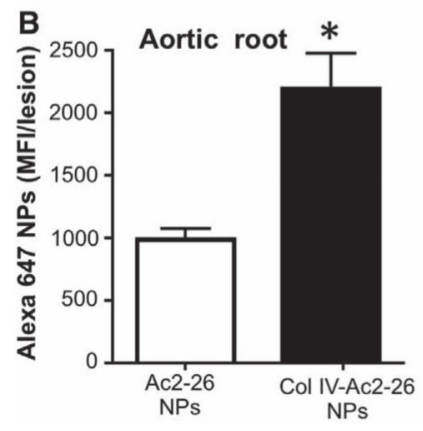
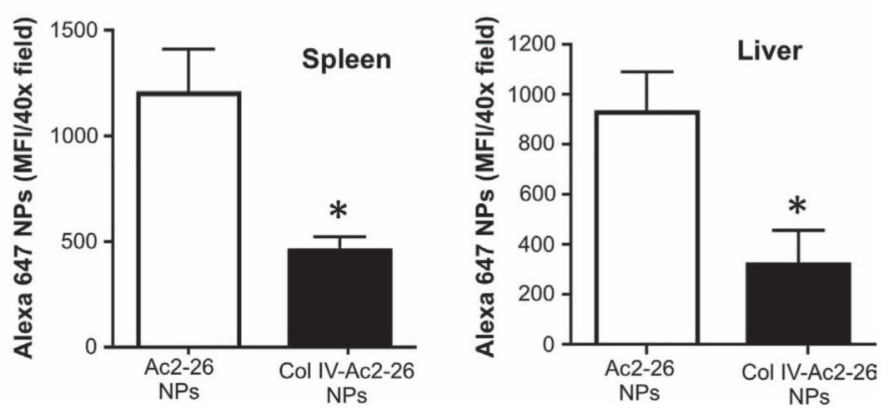

Fig. 1. Col IV Ac2-26 NPs home to atherosclerotic lesions

$\mathrm{Ldlr}^{-/-}$mice were fed a Western diet for 12 weeks and then injected intravenously with Alexa 647-labeled Ac2-26 NPs or Col IV-Ac2-26 NPs. The diet was continued, and aortic root sections were analyzed by fluorescence microscopy 5 days later. (A) Images of 4',6diamidino-2-phenylindole (DAPI)-stained aortic root sections showing NPs (red) and nuclei (blue). The lesions in each image are outlined. To the right of each main image is a zoomedin image of the subendothelial region depicted by the white box. Scale bar, $100 \mu \mathrm{m}$. (B) Sections of aortic root, spleen, and liver (six sections per mouse) were quantified for mean Alexa 647 fluorescence intensity (MFI) using image processing. Data are means \pm SEM $(n=$ 3 separate mice). $* P<0.05$ versus Ac2-26, Student's $t$ test. 
A
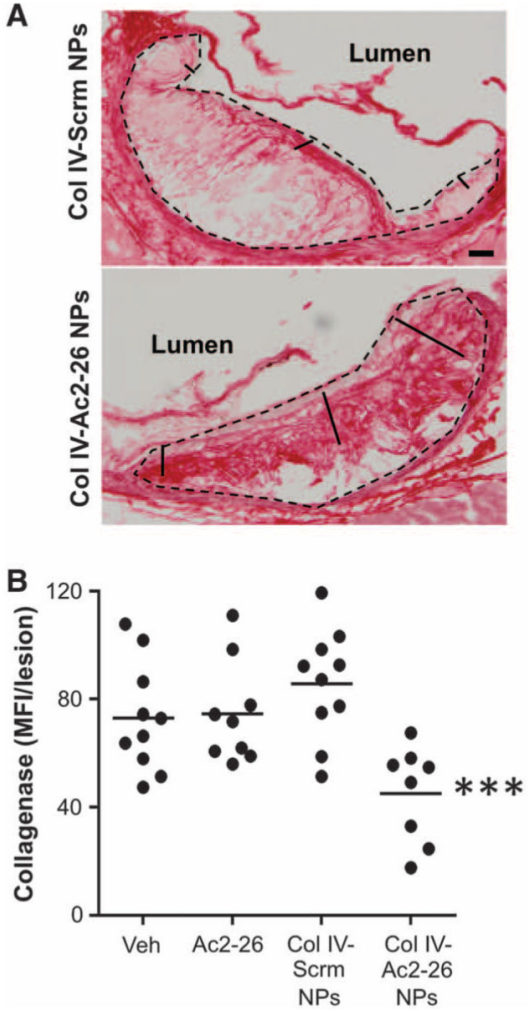

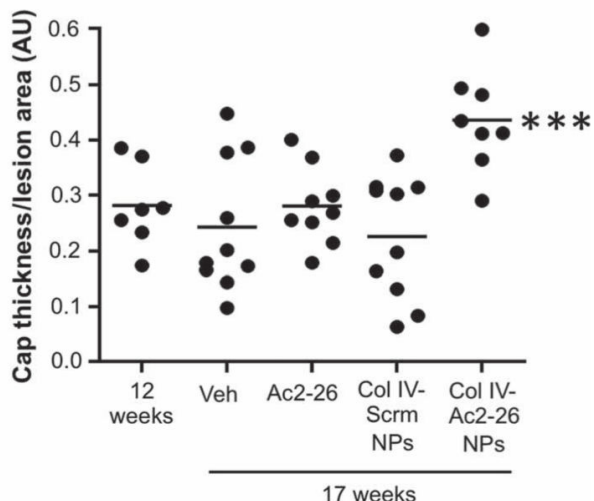

C

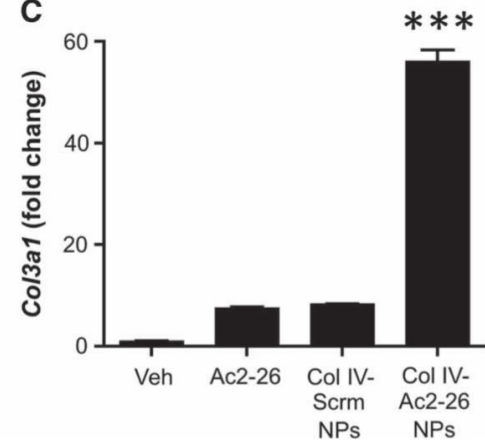

Fig. 2. Col IV-Ac2-26 NPs increase subendothelial collagen in $\mathrm{Ldlr}^{-/-}$mice with established atherosclerosis

$\mathrm{Ldll}^{-1-}$ mice were fed the Western diet for 12 weeks and then injected intravenously with vehicle, free Ac2-26, or Col IV NPs containing scrambled peptide (Scrm) or Ac2-26 once per week for 5 weeks, with the mice remaining on the diet. (A) Aortic root sections from the indicated groups of mice were stained with picrosirius red. The pair of images from the 17week Col IV-Scrm NP and Col IV-Ac2-26 NP cohorts (left) shows examples of measuring lines used to measure cap thickness. The microscopic images were quantified by image processing for fibrous cap thickness/lesion area ratio, expressed as arbitrary ratio units (AU) (right). Data are individual mice, with means shown as horizontal lines $(n=8$ to 10 separate mice, two sections per mouse). Scale bar, $100 \mu \mathrm{m}$. (B) Collagenase activity quantified in aortic root sections using fluorescence microscopy and image processing. Data are individual mice, with means shown as horizontal lines ( $n=8$ to 10 separate mice, two sections per mouse). (C) RNA from the aortic root lesions of five mice per treatment group was obtained by laser capture microdissection (LCM), pooled, and quantified by reverse transcription quantitative polymerase chain reaction (RT-qPCR) for Col3al mRNA, with normalization to lesional Actb mRNA. Data are means \pm SEM. $* * * P<0.001$ versus all other groups, one-way analysis of variance (ANOVA) with post hoc Tukey analysis. 
A

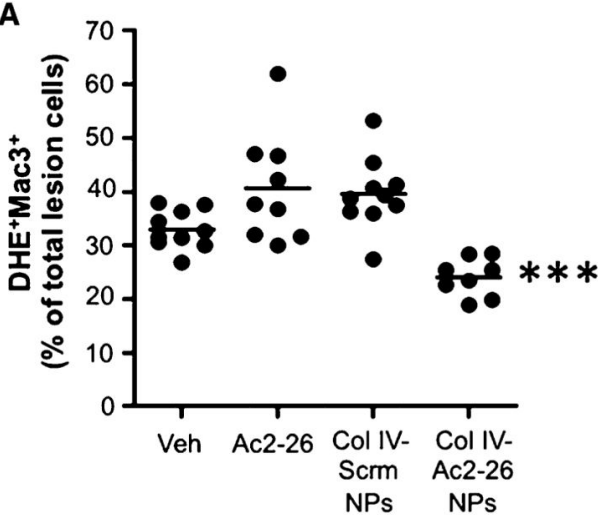

B

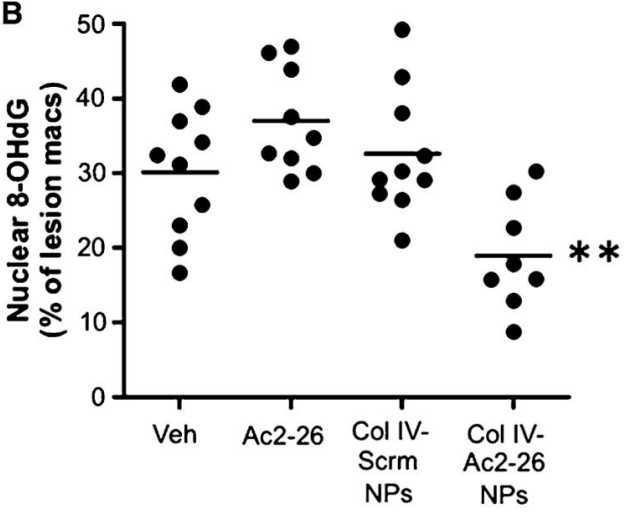

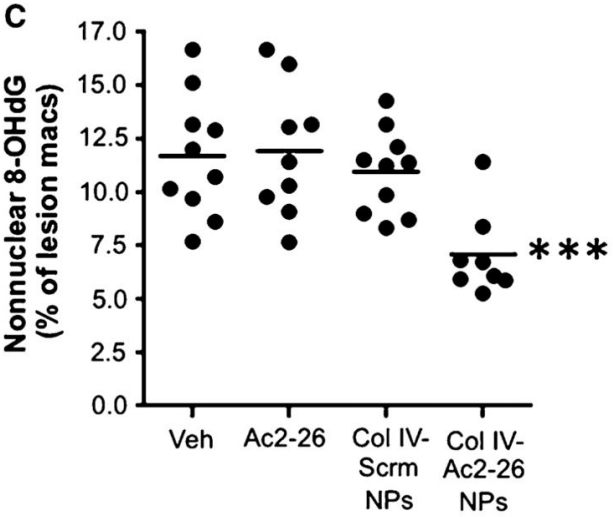

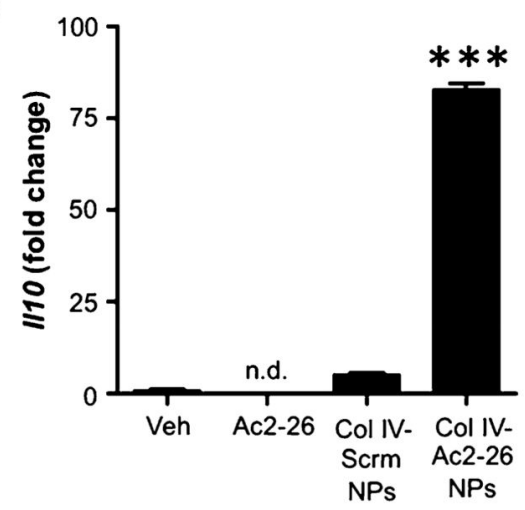

Fig. 3. Col IV-Ac2-26 NPs suppress lesional superoxide and increase lesional $1110 \mathrm{mRNA}$ in $\mathrm{Ldlr}^{-1-}$ mice with established atherosclerosis

(A to C) $L d l r^{-/-}$mice were fed the Western diet for 12 weeks and then injected intravenously with vehicle, free Ac2-26, or Col IV NPs containing scrambled peptide (Scrm) or Ac2-26 once per week for 5 weeks, with the mice remaining on the diet. (A) Superoxide (DHE), macrophages (Mac3), and DAPI nuclear staining were assessed by fluorescence microscopy, and the percent of $\mathrm{DHE}^{+} \mathrm{Mac}^{+}$double-labeled intimal cells per total $\mathrm{DAPI}^{+}$intimal cells was quantified using image processing. Nuclear (B) and nonnuclear (C) 8-OHdG staining in $\mathrm{Mac}^{+}$cells as a percentage of total lesional $\mathrm{Mac}^{+}$cell was quantified by fluorescence microscopy and image processing. Data are individual mice, with means shown as horizontal lines ( $n=8$ to 10 , two sections per mouse). (D) Lesional Il10 mRNA was quantified by RT-qPCR, with normalization to lesional Actb mRNA. Data are means $\pm \operatorname{SEM}\left(n=8\right.$ to 10 separate mice, two sections per mouse). ${ }^{*} * P<0.01, * * * \mathrm{P}<$ 0.001 versus all other groups, one-way ANOVA with post hoc Tukey analysis. 

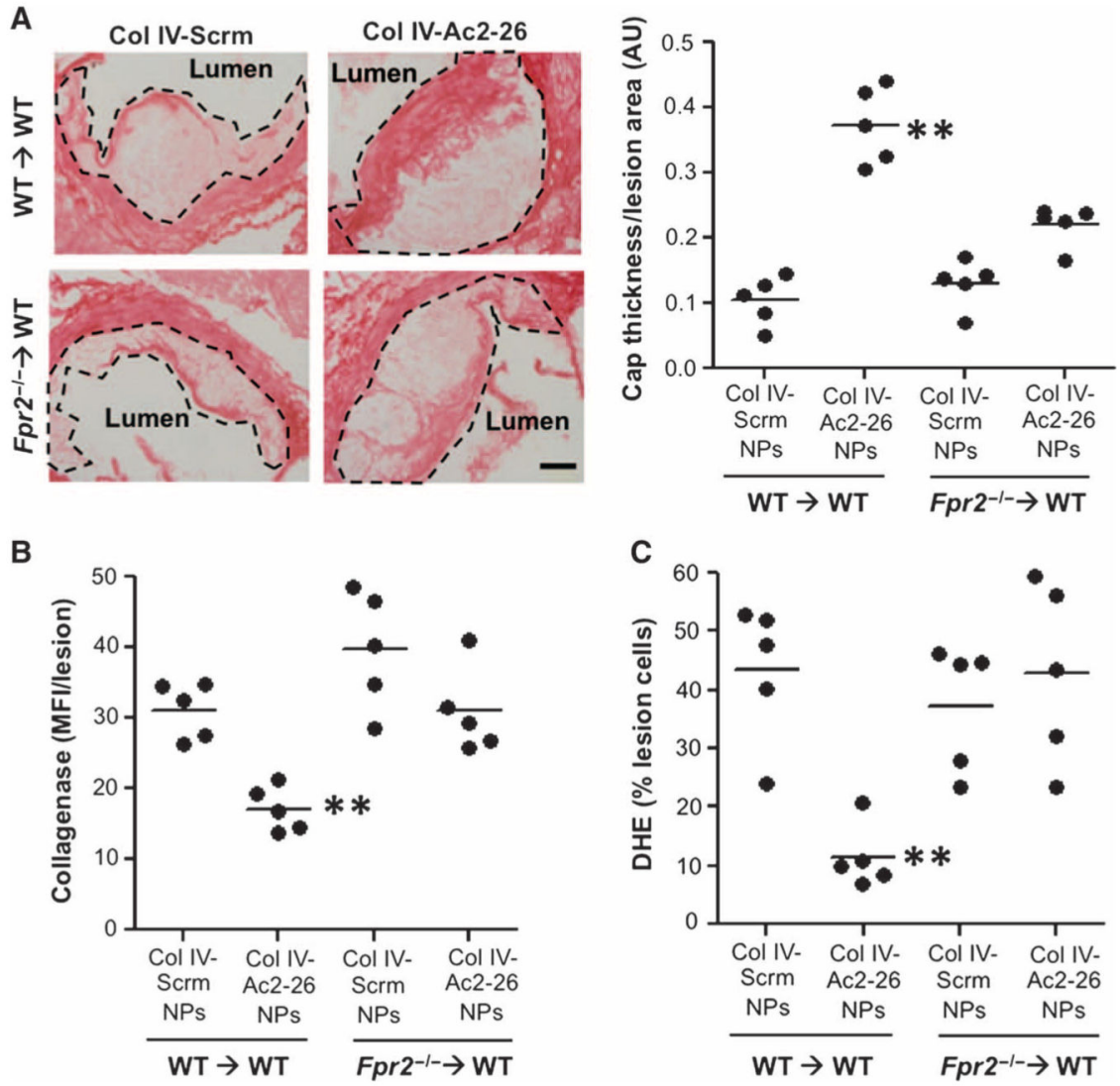

Fig. 4. Col IV-Ac2-26 NPs exert atheroprotective actions in myeloid-derived cells in an FPR2/ ALX-dependent manner

$\mathrm{Ldlr}^{-/-}$mice were transplanted with bone marrow from WT or Fpr2 ${ }^{-/-}$bone marrow. (A) Aortic root sections were stained with hematoxylin and eosin (H\&E) (left), and cap thickness was quantified (right). Scale bar, $100 \mu \mathrm{m}$. (B) Collagenase activity was quantified in aortic root sections using fluorescence microscopy and image processing. (C) Superoxide (DHE) was assessed by fluorescence microscopy. Data are for individual mice, with means shown as horizontal lines ( $n=5$ separate mice, two sections per mouse). $* * P<0.01$ versus all other groups, one-way ANOVA with post hoc Tukey analysis. 

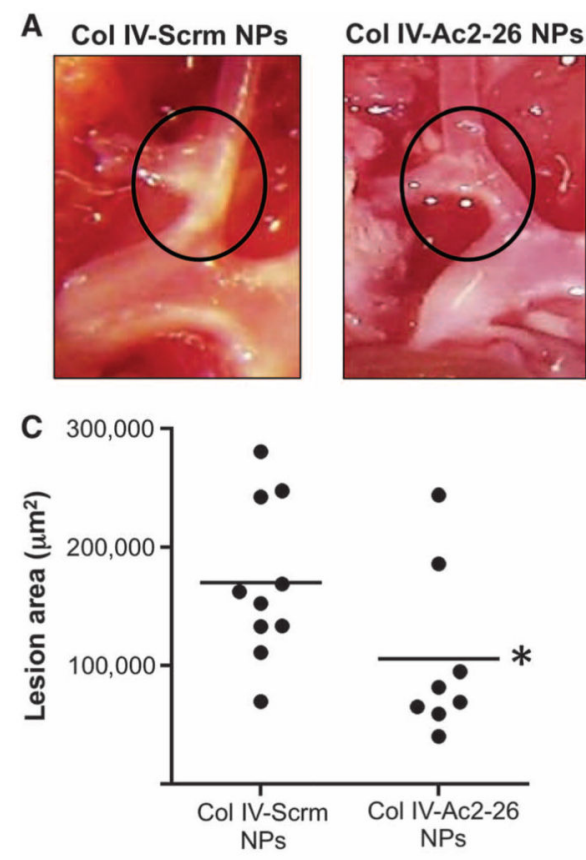

E

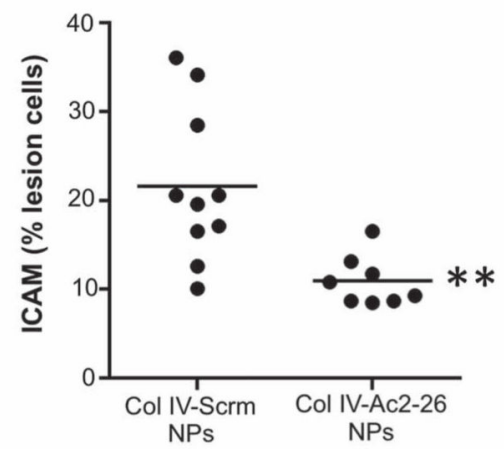

B

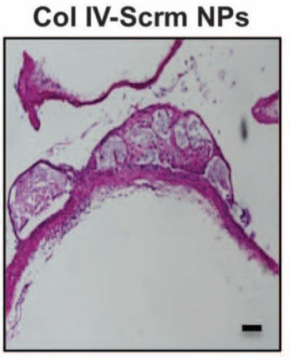

Col IV-Ac2-26 NPs

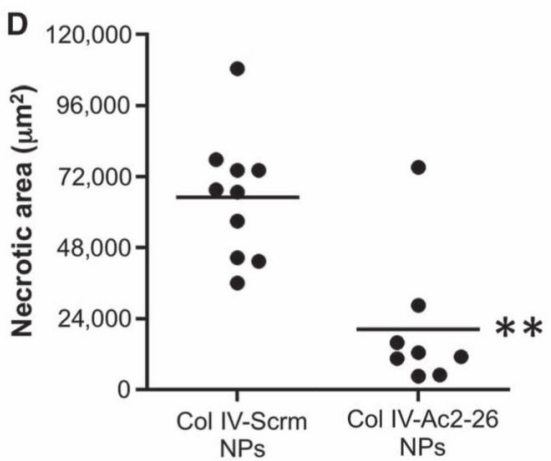

$\mathbf{F}$

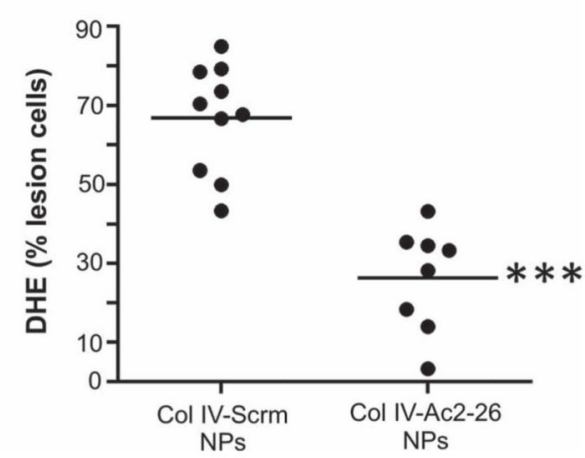

Fig. 5. Col IV-Ac2-26 NPs decrease lesion area, necrotic area, and oxidative stress in brachiocephalic arterial plaques

$\mathrm{Ldlr}^{-/-}$mice were fed the Western diet for 12 weeks and then injected intravenously with Col IV NPs containing scrambled peptide (Scrm) or Ac2-26 once per week for 5 weeks, with the mice remaining on the diet. (A and $\mathbf{B}$ ) Representative whole-mount (A) or crosssectioned specimens (B) of BCA lesions were visualized en bloc or in cross section. Scale bar, $100 \mu \mathrm{m}$. (C to F) Cross sections were quantified for lesion area (C), necrotic area (D), ICAM immunostaining (E), and superoxide (DHE fluorescence) (F). Data are for individual mice, with means shown as horizontal lines ( $n=8$ to 10 separate mice, two sections per mouse). $* P<0.05, * * P<0.01, * * * P<0.001$, Student's $t$ test. 